\title{
AtMospheric Propagation Impairment EFFECTS FOR WIRELESS COMMUNICATIONS
}

\author{
Sharmin Tajria Ananya ${ }^{1}$, Md. Shariful Islam ${ }^{1 *}$, Md.Ashek Raihan Mahmud ${ }^{1}$, \\ Pallab Kanti Podder ${ }^{2}$ and Md Jashim Uddin ${ }^{1}$ \\ ${ }^{1}$ Department of Information and Communication Technology, Islamic University, \\ Kushtia-7003, Bangladesh \\ ${ }^{2}$ Department of Information and Communication Engineering, Pabna University of \\ Science and Technology, Pabna, Bangladesh
}

\begin{abstract}
The prime aspect of this paper is to report the atmospheric impairment effects in wireless communication signalfor different frequencies. Comprehensive tests have been carried out to justify and confront theattenuation impacts of rain, cloud and water vapor oxygen for various wave length channels. The result of the atmospheric impairments is obtained by the parameters which are resembled to the real world measurements. Moreover, the performance is analyzed by the use of different propagation modules and transmission parameters that are frequently used in satellite and radar communications. Experimental resultsindicate that the attenuation effect due to rain has initiated at frequencies above $10 \mathrm{GHz}$ while, other atmospheric effects preceded by attenuation of the cloud and gasses have less effect. It is also observed thatduring frequency transmission, the rate of rainfall and relative humidity become directly proportional to signal quality degradation.
\end{abstract}

\section{KEYWORDS}

Atmospheric Impairment, Different frequencies wireless communications, Satellite communications, Radar communications.

\section{INTRODUCTION}

Radio frequency (RF) corresponds to the spectrum of oscillation of electromagnetic radio waves ranges $3 \mathrm{KHz}$ to $300 \mathrm{GHz}$ and to the alternating current that carries the radio signals. Among these frequency bands, one band is called Super High Frequency (SHF) of frequency range from $3 \mathrm{GHz}$ to $30 \mathrm{GHz}$ and other band is called Extremely High Frequency (EHF) which is called millimeter wave of frequency range from $30 \mathrm{GHz}$ to $300 \mathrm{GHz}$. In wireless communications the radio frequency signal radiates from the transmitting antenna to the receiving antenna through a space communication link. The quality of the signal deteriorates as it propagates through the link due to the particles absorption and scattering in space[1-4]. Theindicated declination of the transmitted signal remarkably affects the receiving end data especially in the modern satellite technology that needed a high data rate. Fast and slow fluctuations of signal result in atmospheric event [5]. In earlier is called scintillation, lead to rapid signal output fluctuations forced on the medium to the unregulated refractive index inhomogeneity. Meanwhile, slow fluctuations are generally caused by the particles absorbing and dispersing the signal energy, primarily water droplets in the satellite-earth station link. Ionospheric scintillation and tropospheric scintillation occurs due to the non-uniformities in electron density in the atmosphere as well as asymmetry in 
radio refractivity as the wave propagates along various medium densities in the troposphere [6]. The frequency, ' $\mathrm{f}$ ' and the angle of elevation ' $\theta$ ' are the transmitted signal parameters changed for that have been changed because of the major impact on the amount of impairments in atmosphere. The ionospheric scintillation has a great effect on less than $3 \mathrm{GHz}$ transmitted signal. However, this effect decreases with increasing the frequency [7]. For frequencies above $10 \mathrm{GHz}$, the rest of the phenomena, like rain, cloud attributed a major effect on signal attenuation [8]. Contrarily, oxygen and water vapor particles in space have a huge impact on high frequency signal bands. [9].Transmission in the rain at low elevation angles, condensed clouds, water vapor and oxygen can perk up the effective rain, clouds, water vapor as well as oxygen in the medium signal conductions, respectively, which in turn will dilapidated the amount of signal obtained. So, there is a strong relation between elevation angle and earth station to reduce the influence of atmospheric parameters. It is essential to develop a good prediction model for increasing the earth sky wireless communication quality to index the level of atmospheric fade and the selection of the appropriate fade mitigation technique (FMT).

\section{MATerials AND Methods:}

\section{Channel Model}

There are three main parts in the general satellite wireless communication system model, namely: Earth station, Satellite and the link between earth station and satellite. The channel and receiver model is made by using MATLAB based on the radio wave recommended by International Telecommunication Union (ITU) and demonstrated that suitable for wireless communications, especially in satellite communication. The link of the satellite is affected by atmospheric impairments which are demonstrated subsequently through this paper.

\section{ATTENUATION FOR RAIN}

The transmitted signal power is attenuated by rain due to the absorption and dispersion of the signal energy depending on the form, size and quantity of droplets that the signal passes through the medium and rain rate[9]. The effect of shape and size of the rain drop is shown in Fig.1.

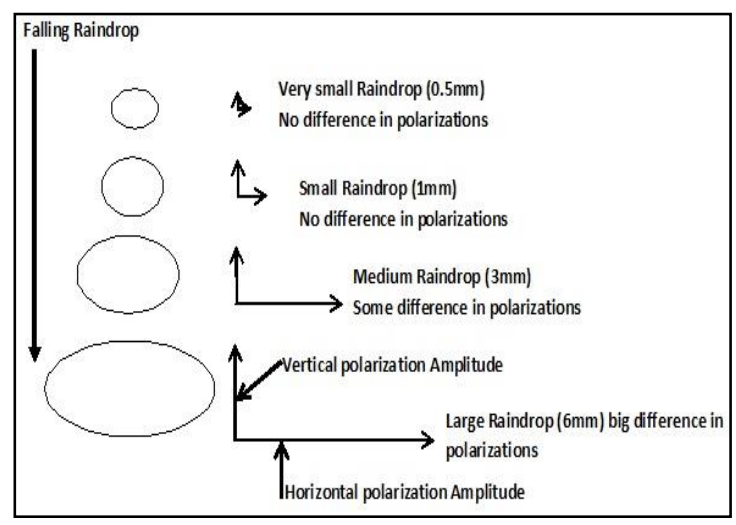

Fig.1 Raindrop size and shape

Rain typically occurs at different altitudes above sea level depending on a region of the earth. Some state of the art rain attenuation prediction model have been published by the experienced researcher which maintain world agreements such as COST 255 [10], Crane [11], a group of research team from International Telecommunication Union Radio wave sector (ITU-R) [12, 13], 
DAH [14], and SAM[15]. However, another model of rain attenuation shown in Fig.2 is based on updated ITU-R prediction model.

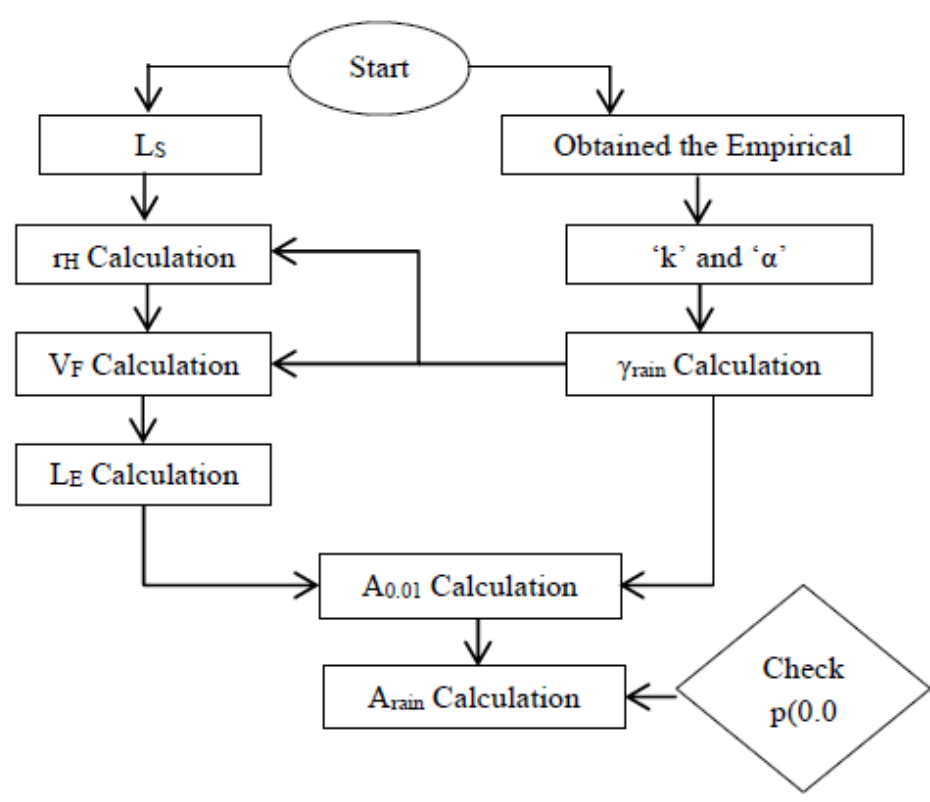

Fig.2 Rain attenuation model

Especially, the concrete calculated rain rate in [9], somewhat the predicted values by the ITU-R model, be appropriated for construction a reliable estimation model that was implemented by using MATLAB. The starting point in the flowchart of the rain attenuation model contains the values of the earth station position parameters such as latitude as well as height above sea level and rain parameters such as rain rate, rain height, percentage of overtime or exceedance time ' $p$ ' with some parameters for the transmitter, like frequency ' $\mathrm{f}$ ' elevation angle ' $\theta$ ' and polarization angle ' $\tau$ '. The rain specific coefficients ' $k_{s}$ ' and ' $\alpha_{s}$ ' values are obtained by calculating through Eq. (1) and Eq. (2).

$$
\begin{gathered}
k_{s}=\frac{k_{H}+k_{V}+\left(k_{H}-k_{V}\right) \cos ^{2} \theta \cos (2 \tau)}{2}(1) \\
\alpha_{s}=\frac{k_{H} \alpha_{H}+k_{V} \alpha_{V}+\left(k_{H} \alpha_{H}-k_{V} \alpha_{V}\right) \cos ^{2} \theta \cos (2 \tau)}{2 k}(2)
\end{gathered}
$$

The specific attenuation of the rain (rain attenuation $/ 1 \mathrm{Km}$ ) is then calculated using Eq. (3) based on the actual measured rainfall rate (at $\mathrm{p}=0.01 \%$ ) [9] rather than ITU predicted value [13].

$$
\gamma_{\text {rain }}=\alpha_{s}\left(R_{0.01}\right)^{k_{s}}(3)
$$

This value will be used for identifying the effective path length and for predicting the overall rain attenuation. The horizontal reduction factor $\left(r_{H O R}\right)$ is determined for $0.01 \%$ of the time by Eq. (4).

$$
r_{\text {HOR }}=\frac{1}{1+0.78 \sqrt{\left(\frac{P_{H} \gamma_{R}}{f}-0.38\left(1-e^{-2 P_{H}}\right)\right)}}(4)
$$


where $P_{H}$ is the horizontal projection which is dependent to slant path length ' $L_{S l}$ ' and the elevation angle ' $\theta$ ' calculated by Eq. (5).

$$
P_{H}=L_{S l} \cos \theta(5)
$$

The slant path length varies on the vertical height from the earth station to the height of rain and " $\theta$ ' calculated by Eq. (6).

$$
L_{S l}=\left\{\begin{array}{cc}
\frac{H_{\text {rain }}-H_{e s}}{\sin \theta} & \text { for } \theta \geq 5^{\circ} \\
\frac{2\left(H_{\text {rain }}-H_{e s}\right)}{\sin ^{2} \theta+\frac{2\left(H_{\text {rain }}-H_{e s}\right)}{E_{e r}}+\sin \theta} & \text { for } \theta<5^{\circ}
\end{array}\right.
$$

where $H_{\text {rain }}$ and $H_{e s}$ are the heights of rain and earth station above sea level, respectively; and $E_{e r}$ is indicating the radius of earth $\left(85 \times 10^{5} \mathrm{~m}\right)$. The vertical fitting or adjustment factor $\left(V_{F}\right)$ calculated at $0.01 \%$ of the time using Eqs. (7) to (9).

$$
\begin{gathered}
\xi=\tan ^{-1}\left(\frac{H_{\text {rain }}-H_{e s}}{P_{H} \gamma_{H}}\right)(7) \\
L_{R}=\left\{\begin{array}{cc}
\frac{P_{H} \gamma_{H}}{\cos \theta} \quad \text { for } \xi>\theta \\
\frac{H_{\text {rain }}-H_{e s}}{\sin \theta} \quad \text { for } \xi \leq \theta
\end{array}\right. \\
V_{F}=\frac{1}{1+\sqrt{\sin \theta}\left[31\left(1-e^{-\frac{\theta}{1-x}}\right) \frac{\sqrt{L_{R} \gamma_{R}}}{f^{2}}-0.45\right]}(9)
\end{gathered}
$$

where the variable ' $x$ ' is depended on the earth station latitude $(\varphi)$. In ITU-R model the calculation of the horizontal and vertical adjustment factors is based on $0.01 \%$ of the time exeedance due to these factors are the indication oftemporal variation for the rain drop dimension and rain height, respectively[16]. The effective path length and thetotal rain attenuation can be calculated at $0.01 \%$ of time $\left(A_{0.01}\right)$ by Eqs. (10) and (11).

$$
\begin{gathered}
L_{E}=L_{R} V_{F}(10) \\
A_{0.01}=L_{E} \gamma_{R}(11)
\end{gathered}
$$

The foreseen attenuation of rain at any percentage of time ( $p$ ) can be calculated using Eqs. (12) and (13)

$$
\begin{gathered}
\beta=\left\{\begin{array}{cc}
0 & i f p \geq 1 \% \text { or }|\varphi| \geq 36^{\circ} \\
-0.005(|\varphi|-36) & \text { ifp }<1 \% \text { and }|\varphi|<36^{\circ} \text { and } \theta \geq 25^{\circ} \\
-0.005(|\varphi|-36)+1.8-4.25 \sin \theta & \text { Otherwise }
\end{array}\right. \\
A_{\text {rain }}=A_{0.01}\left(\frac{p}{0.01}\right)^{-\left[0.655+0.033 \ln (p)-0.045 \ln \left(A_{0.01}\right)-\beta(1-p) \sin \theta\right]}
\end{gathered}
$$

Signal performance is analyzed at different transmission parameters for the period of rain occurrences, the signal strength as well as error rate assessments received. 


\section{Attenuation Due to Cloud}

One of the cloud definition is given by World Meteorological Organization (WMO) as an amount of very small droplets of water (above $0^{\circ} \mathrm{C}$ ), ice crystals (between $-20^{\circ} \mathrm{C}$ and $-40^{\circ} \mathrm{C}$ ) or a maximum of both whose presence is observed primarily in the troposphere layer at various heights above ground level [17]. A state of the art classification of cloud is shown in Table.1 [18].

Table 1: Classification of cloud

\begin{tabular}{|c|c|c|c|c|}
\hline $\begin{array}{c}\text { Cloud } \\
\text { Generation }\end{array}$ & Cloud type & $\begin{array}{c}\text { Cloud base } \\
(\mathrm{km})\end{array}$ & $\begin{array}{c}\text { Liquid water content } \\
\text { LWC }\left(\mathrm{g} / \mathrm{m}^{3}\right)\end{array}$ & $\begin{array}{c}\text { Vertical thickness } \\
(\mathrm{km})\end{array}$ \\
\hline \multirow{3}{*}{ High clouds } & Cirrus & $6-12$ & Non-available & Non-available \\
\cline { 2 - 5 } & Cirrocumulus & $6-12$ & Non-available & Non-available \\
\cline { 2 - 5 } & Cirrostratus & $6-12$ & 0.10 & Non-available \\
\hline \multirow{3}{*}{ Middle clouds } & Altocumulus & $2-6$ & 0.20 & Non-available \\
\cline { 2 - 5 } & Altostratus & $2-6$ & 0.41 & 0.5 \\
\cline { 2 - 5 } & Nimbostratus & Ground-3 & 0.61 & 0.8 \\
\hline \multirow{3}{*}{ Low clouds } & Stratocumulus & $0.3-1.350$ & 0.30 & 0.5 \\
\cline { 2 - 5 } & Stratus & Ground-0.6 & 0.42 & 2.0 \\
\cline { 2 - 5 } & Cumulus & $0.3-1.5$ & 1.00 & 3.0 \\
\cline { 2 - 5 } & Cumulonimbus & $0.6-1.5$ & 0.51 & \\
\hline
\end{tabular}

Electromagnetic energy also absorbed and scattered by the cloud liquid water content (LWC) especially for frequencies above $10 \mathrm{GHz}$, but impact on the propagated signal is less strength than rain [9]. Cloud attenuation due to cloud depends on some parameters in transmission, for instance, the frequency of the signal, the angle of elevation ' $\theta$ ' as well as cloud parameters, for instance, average height and thickness, total columnar content of liquid water in $g / \mathrm{m}^{3}$ (LWC) and temperature. To obtained attenuation due to cloud, a number of well accepted models has been developed by some experienced researchers, like Salonen, Uppala[19], ITU-R [20], DAH [21] and Altshuler, Marr [22]. In Salonen, Uppala and ITU-R cloud attenuation prediction models are both in identical but difference only in LWC is shown in Fig.3.

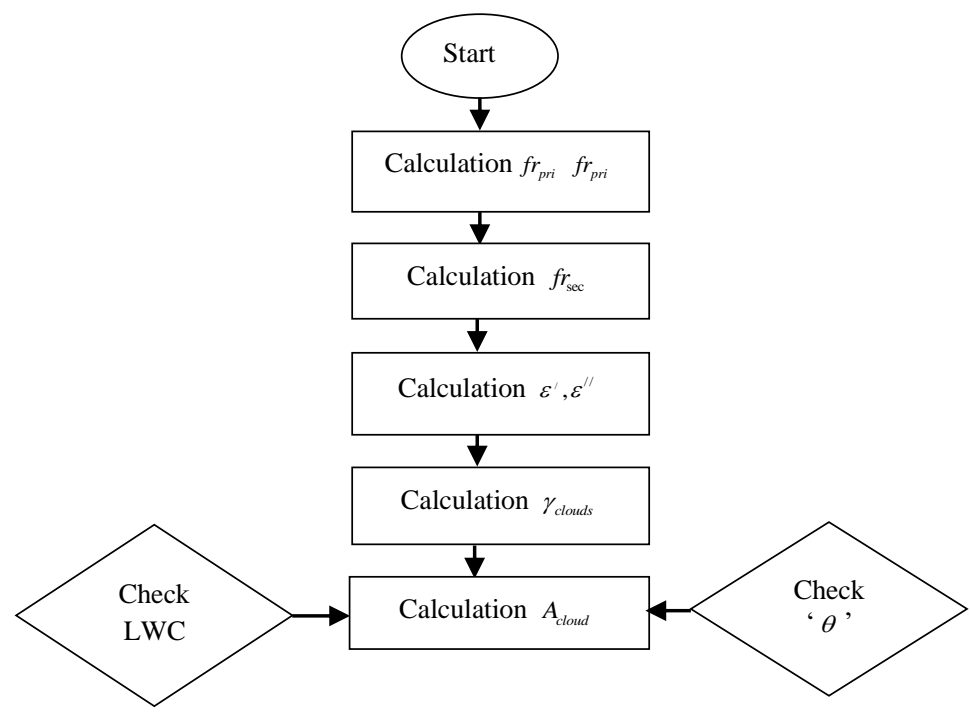

Fig. 3: Cloud attenuation model flowchart 
In this implementation of this model is started with start that contains the parameters already stated in earlier. The principal and secondary relaxation frequencies $f r_{p r i}$ and $f r_{\text {sec }}$ respectively, are calculated by the Eqs. (14) and (15).

$$
\begin{gathered}
f r_{p r i}=20.09-142(\Lambda-1)+294(\Lambda-1)^{2}(14) \\
f r_{\mathrm{sec}}=590-1500(\Lambda-1)(15)
\end{gathered}
$$

where $\Lambda=300 / T_{k}$, where $T_{k}$ is the temperature, measured in Kelvin. The Eqs. (16) and (17) describe the complex dielectric permittivity of water contents within the cloud.

$$
\begin{gathered}
\varepsilon^{\prime}=\frac{\varepsilon_{0}-\varepsilon_{1}}{1+\left(\frac{f}{f r_{p r i}}\right)^{2}}+\frac{\varepsilon_{1}-\varepsilon_{2}}{\left(\frac{f}{f r_{\mathrm{sec}}}\right)^{2}}+\varepsilon_{2}(16) \\
\varepsilon^{\prime \prime}=\frac{f\left(\varepsilon_{0}-\varepsilon_{1}\right)}{f r_{p r i}\left[1+\left(\frac{f}{f r_{p r i}}\right)^{2}\right]}+\frac{f\left(\varepsilon_{1}-\varepsilon_{2}\right)}{f r_{\mathrm{sec}}\left[1+\left(\frac{f}{f r_{\mathrm{sec}}}\right)^{2}\right]} \text { (17) }
\end{gathered}
$$

where $\varepsilon_{0}=77.6+103.3(\Lambda-1)$ and $\varepsilon_{1}, \varepsilon_{2}$ values are 5.48 and 3.51 , respectively. It can be calculated the cloud specific attenuation coefficient by Eq. (18)

$$
\gamma_{\text {clouds }}=\frac{0.819 f}{\varepsilon^{\prime \prime}\left[1-\left(\frac{2+\varepsilon^{\prime}}{\varepsilon^{\prime \prime}}\right)^{2}\right]}(18)
$$

A certain value of probability of the cloud attenuation depends on LWC that can be obtained for the region of interest (ROI) from radiometric measurements.

$$
A_{\text {clouds }}=\gamma_{\text {clouds }}\left(\frac{L W C}{\sin \theta}\right)(19)
$$

On the other hand, the $L W C$ can be estimated using either the expected values of Salonen, Uppala [19] or ITU-R study group 3 [20]. Preliminary, it is assumed that $L W C$ is obtained from the proposed diagram depending on the cloud base temperature and height. The later implies that the use of exceeded annual values for a number of percentages of annual time from the digital maps they proposed containing values at some specific locations. By using interpolation, it can be derived for other desired location on Earth

\section{WATER VAPOR AND OXYGEN AtTENUATION}

Owing to the presence of water vapor and dry air ingredients in the wireless transmission medium, the electromagnetic wave propagation through the atmosphere suffers signal quality degradation[23]. Some significant parameters are dependent on water vapor attenuation as for example temperature, water vapor content, and altitude above sea level. There is a relation among the attenuation due to water, temperature and relative humidity $(\mathrm{RH})$ that is attenuation rises proportionally when increases temperature and comparative humidity. On the other hand, oxygen has the significant impact between all other gases owing to contain $20.94 \%$ oxygen in the dry atmosphere. So, there is a significant impact on satellite wireless communications frequencies above $50 \mathrm{GHz}[6,24]$. The attenuation due to oxygen is differed by other gaseous 
ingredients of the atmospheric disorders because the effect remains constant and distinct on all the regions of the earth. The renowned researchers carried out several experiments using radiosonde or the purpose of findings and predicting the water content as well as attenuation of oxygen [24, 25].However, the propagation sector of ITU-R developed a prediction model [26, 27] which has been accepted in global agreements. Water vapor and oxygen attenuation prediction model flowchart shown in Fig. 4 that has been introduced based on ITU-R estimation prediction model was started with allied parameters for instance, frequency that is intended to transmit, and relative humidity. Mean temperature as well as pressure.

It can be determined oxygen specific attenuation for frequencies up to $350 \mathrm{GHz}$ by the series of equations where frequency ranges are- $f_{0} \leq 54,64<f_{0} \leq 60,60<f_{0} \leq 62,62<f_{0} \leq 66,66<f_{0} \leq 120,120<f_{0} \leq 350$

$$
\gamma_{o}=f_{0}^{2} r_{p}^{2} r_{T}^{3.5}\left[\frac{3.02 \times 10^{-4}}{1+1.9 \times 10^{-5} f_{0}^{1.5}}+\frac{0.283 r_{T}^{0.3}}{\left(f_{0}-118.75\right)^{2}+2.91 r_{p}^{2} r_{T}^{1.6}}\right] \times 10^{-3}+\partial \text { when } 120<f_{0} \leq 350(20)
$$

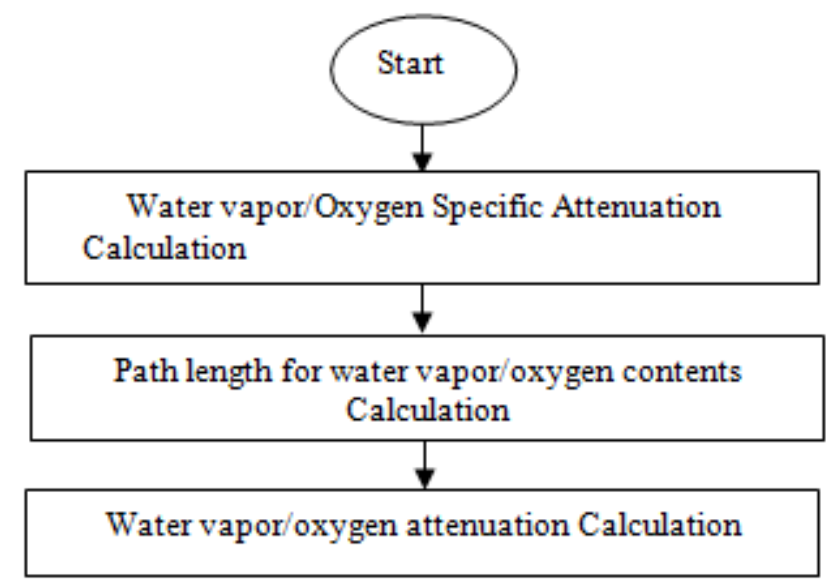

Fig.4 Water vapor or Oxygen attenuation model

where Pressure ( $r_{p}=$ Pressure/1013) and temperature $\left(r_{T}=288 / \mathrm{T}\right)$ respectively. The value of $\partial$ can be obtained ${ }^{[26]}$. The path length for oxygen content can be found using Eq. (21).

$$
\begin{gathered}
L_{O}=\frac{6.1\left(1+m_{1}+m_{2}+m_{3}\right)}{1+0.17 r_{p}^{-1.1}}(21) \\
\text { where } m_{1}=\frac{4.64}{1+0.066 r_{p}^{-2.3}} \exp \left[-\left(\frac{f-59.7}{2.87+12.4 \exp \left(-7.9 r_{p}\right)}\right)^{2}\right] m_{2}=\frac{0.14 \exp \left(2.12 r_{p}\right)}{\left(f_{0}-118.75\right)^{2}+0.031 \exp \left(2.2 r_{p}\right)} \text { and } \\
m_{3}=\frac{0.0114 f_{0}}{1+0.14 r_{p}^{-2.6}}\left(\frac{-0.0247+0.0001 f_{0}+1.61 \times 10^{-6} f_{0}^{2}}{1-0.0169 f_{0}+4.1 \times 10^{-5} f_{0}{ }^{2}+3.2 \times 10^{-7} f_{0}^{3}}\right)
\end{gathered}
$$

Presently, it can be calculated of water vapor specific attenuation in $(\mathrm{dB} / \mathrm{km})$ using Eq. (22).

$\gamma_{W}=f_{0}^{2} r_{T}^{2.5} \rho\left[s_{1}+s_{2}+s_{3}+s_{4}+s_{5}+s_{6}+s_{7}+s_{8}+s_{9}\right] \times 10^{-4}$

where $s_{1} t o s_{9}$ values can be found from ${ }^{[26]}$.Some parameters, such as season, latitude and climate change, can vary the effective path length. However, the value of effective water vapor path length in the troposphere can be calculated for $f_{0} \leq 350 \mathrm{GHz}$ using Eq. (23). 


$$
L_{v}=1.66\left(1+\frac{1.39 \sigma_{w}}{\left(f_{0}-22.235\right)^{2}+2.56 \sigma_{w}}+\frac{3.37 \sigma_{w}}{\left(f_{0}-183.31\right)^{2}+4.69 \sigma_{w}}+\frac{1.58 \sigma_{w}}{\left(f_{0}-325.1\right)^{2}+2.89 \sigma_{w}}\right)(
$$

where $\sigma_{W}=\frac{1.013}{1+\exp \left(4.902-8.6 r_{p}\right)}$ Finally, it can be predicted the total gases attenuation $A_{\text {Gases }}$ (Attenuation to the oxygen and water vapor) using Eq. (24).

$$
A_{\text {Gases }}=\frac{A_{O}+A_{W}}{\sin \theta}=\frac{\gamma_{O} L_{O}+\gamma_{W} L_{W}}{\sin \theta}
$$

In order to offer the best possible fade mitigation technique (FMT), another additional model is applied to improve the quality of the signal assessment in wireless communications of satellite networks for variousmodulation types. There are two types of link losses is composed: one is the free space loss (FSL) and other is atmospheric losses. The free space loss is obtained by using the Eq. (25)

$$
F S L=20 \log \left(\frac{4 \pi d}{\lambda}\right)(25)
$$

where $f=\frac{3 \times 10^{8}}{\lambda}$. To analyze the communication signal quality on the basis of the Friis transmission equation, the bit energy to noise ratio $\left(\frac{E_{b}}{N_{o}}\right)$ can be calculated by using Eq. (26)

$$
\frac{E_{b}}{N_{o}}(d B)=E I R P+G_{r}-F S L-L_{A}-L_{S}-N_{0}-10 \log \left(R_{b}\right)(26)
$$

where EIRP is the effective isotropic radiated power, $L_{A}$ is the atmospheric losses, $L_{S}$ is the system losses and $R_{b}$ is the noise spectral density. Important parameter BER degradation is attributed to atmospheric impairments can be found by using Gray code Eq. (27)

$$
B E R \approx \frac{S E R}{\log _{2}(M)}(27)
$$

where the symbol error rate (SER) is obtained by calculating using equations listed in ${ }^{[9]}$ for the modulation techniques QPSK, M-array PSK. The extra model has added to improve the quality of the signal assessment in wireless communications of satellite networks for different modulation schemes already mentioned to propose the optimal FMT. Now a days, satellite wireless communication technologies made a huge using resource management techniques, Channel State Reporting (CSR) and FMT. CSR indexing calculation is found as a quantized and experienced scaled test $E_{b} / N_{0}, E_{s} / N_{0}$, BER and SER. The identified values are very much important for making decision regarding the allocation of resources to users with specific channel conditions or the evaluation of a certain FMT. The transmission time interval (TTI) is applied in TDM/TDMA transmitters. The classification of FMT and their uses in detail reported in ${ }^{[9]}$. The power control technique for uplink and downlink and ground station of a satellite wireless communication can be found in detail [28, 29].

\section{Complete Propagation Model}

A state of the art propagation model for wireless communication in satellite network as shown in Fig.5, consisting of three components: transmitter, channel and receiver. The packet data units (PDU) are transferred using specific transmitting variables as per the underlying propagation model, and the mitigation technique is selected via the reported satellite channel. To observe for proper calculation considering the impact of FSL, dry air (oxygen) and water vapor attenuation are included before the module of cloud attenuation. 
International Journal of Wireless \& Mobile Networks (IJWMN) Vol. 12, No. 3, June 2020

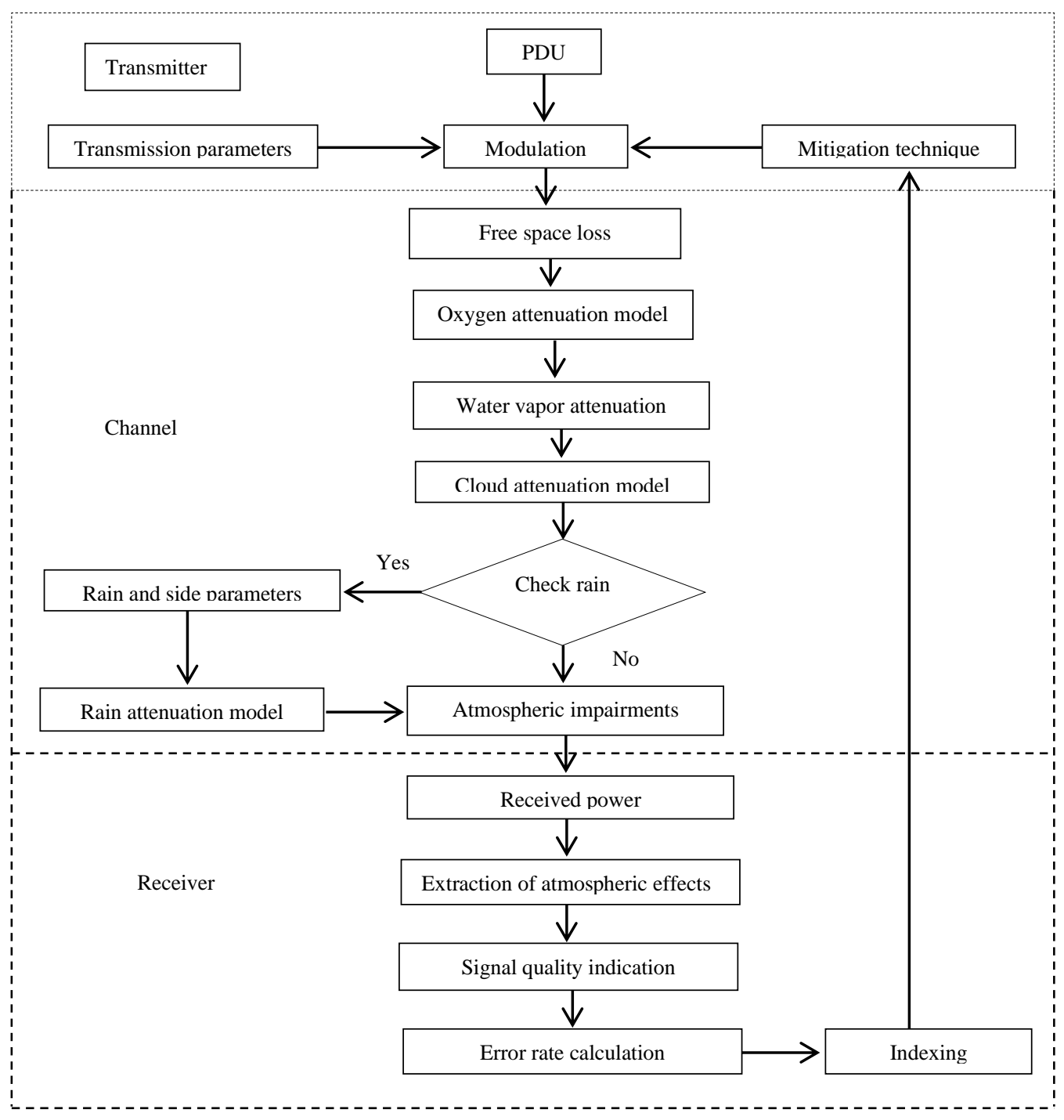

Fig.5 A state of the art propagation model

Attenuation due to rain is then checked for the easy of use. The rain and positional variables are then recognized for formative purposes as having the effect of rain on the signal power. After that, for evaluating the received signal power, total atmospheric impairments are calculated. The effect of atmospheric impairments on the received signal is signified by $E_{b} / N_{0}$ and then examined for evaluation with different elevation angles. To identify the signal quality indication $E_{b} / N_{0}$ is used and BER evaluation with M-array modulation schemes. For processing to estimate the satellite channel quality, the obtained values are then indexed. Then, all values are used to report the transmitter to the next TTI for selecting the correct FMT. Using various parameters relating atmospheric and signal transmission, this propagation model has applied and obtained results for specific modules with signal detection and evaluation.

\section{RESULT AND DISCUSSION}

A major impact on signal strength attenuated during rain events for the frequency of the transmitted signal of a satellite shown in Fig.6. For analyzing, a satellite terminal is considered 
located in Gazipur, Bangladesh (Bongobondhu satellite Lattitude 23.99N, Longitude 90.42E) and the elevation angle ' $\theta$ ' was fixed to $45^{\circ}$.

It is also noticeable by the figure that the $6 \mathrm{GHz} \mathrm{C}$-band transmitted frequency shown to have a small amount of attenuation at heavy rain events ( $0.01 \%$ of time) because this attenuation increases much when transmitted carrier signal frequencies are $11.6 \mathrm{GHz}$ and $22 \mathrm{GHz}$ for $\mathrm{Ku}-$ band as well as Ka-band, respectively.

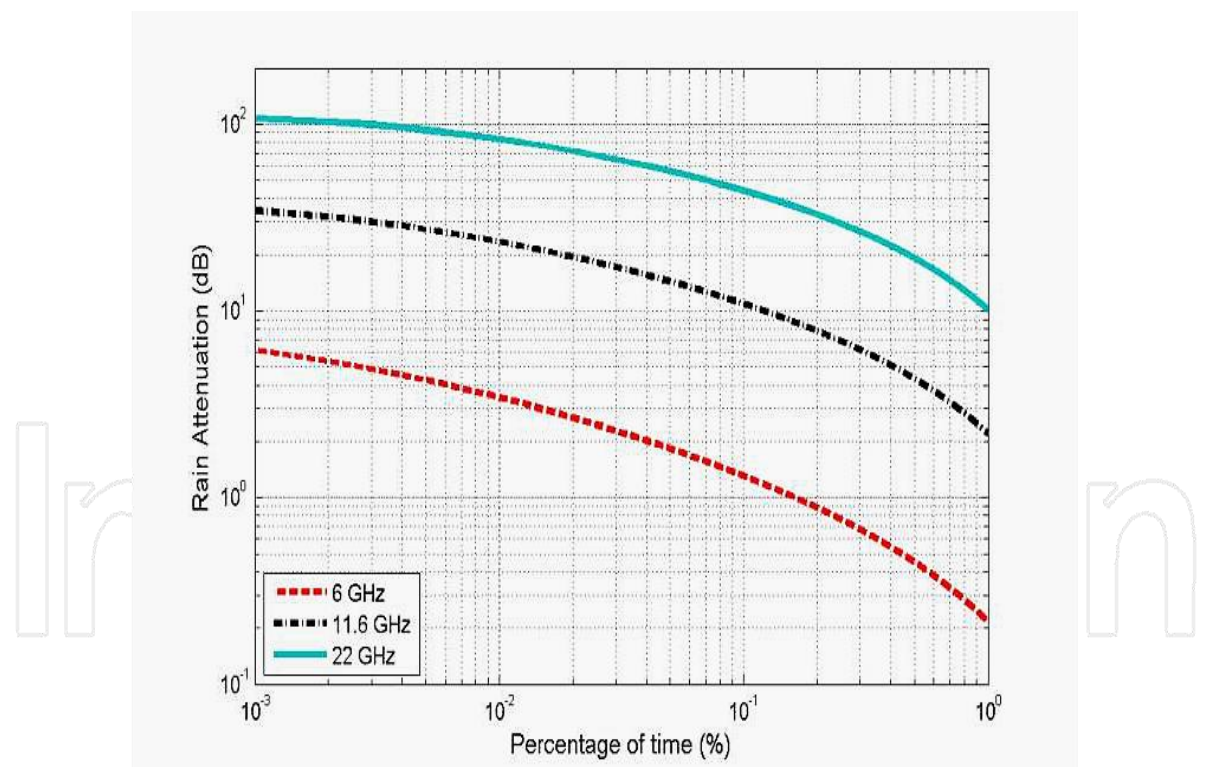

Fig.6 Attenuation due to rain at different percentages of time and frequencies [9]

The specific attenuation $(\mathrm{dB} / \mathrm{km})$ due to atmospheric gases for the frequencies ranges from $1 \mathrm{GHz}$ to $1000 \mathrm{GHz}$ by considering the standard atmospheric conditions, i.e., $\mathrm{T}=293 \mathrm{~K}, \mathrm{P}=101.300 \mathrm{kPa}$, water vapor density, $\rho=7.5 \mathrm{~g} / \mathrm{m}^{3}$ and path distance $1.5 \mathrm{~km}$ is shown the spectrum with dry air (zero water vapor density) in Fig.7. This Figure also reveals that the effect of water vapor (blue curve) is prime dominant over the full spectral range compare than the effect of dry air (red curve). Signal attenuation due to rainfall is shown in Fig.8. When rain rate $1.0 \mathrm{~mm} / \mathrm{h}$ (considered as light rainfall), signal path distance $10 \mathrm{~km}$ and transmitted signal frequency $20 \mathrm{GHz}$ then signal attenuation value is obtained $0.7104 \mathrm{~dB}$ whereas $7.8413 \mathrm{~dB}$ is obtained by applying the parameters, rain rate $10.00 \mathrm{~mm} / \mathrm{h}$ (considered as heavy rainfall), signal path distance $10 \mathrm{~km}$ and transmitted signal frequency $20 \mathrm{GHz}$. 
International Journal of Wireless \& Mobile Networks (IJWMN) Vol. 12, No. 3, June 2020

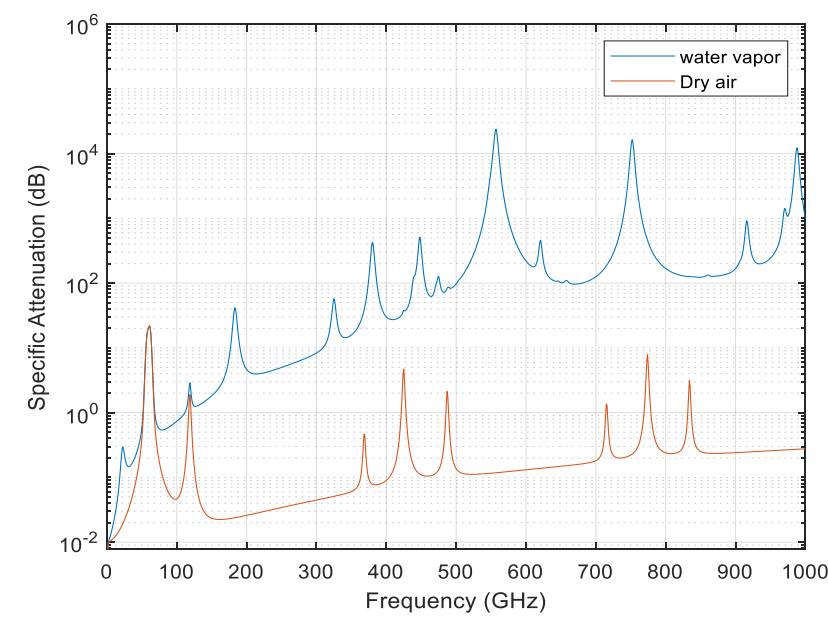

Fig.7 Specific attenuation due to atmospheric gases

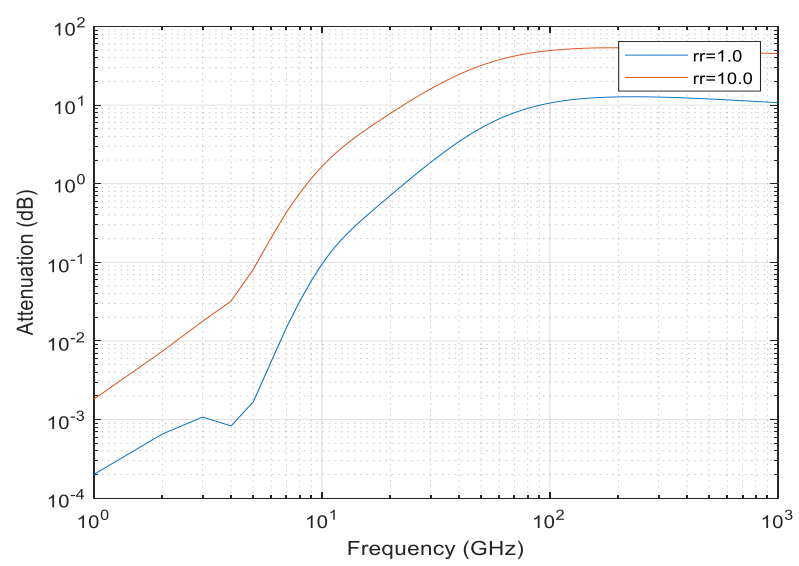

Fig.8 Signal attenuation due to rainfall

The path elevation angle is the parameter for the signal quality degradation. Fig.9 and Fig.10 shows the $100 \mathrm{GHz}$ transmitted signal frequency is attenuated with different path angles where path distance is applied $100 \mathrm{~km}$, rain rates are $10.00 \mathrm{~mm} / \mathrm{h}$ and $1.0 \mathrm{~mm} / \mathrm{h}$ respectively.

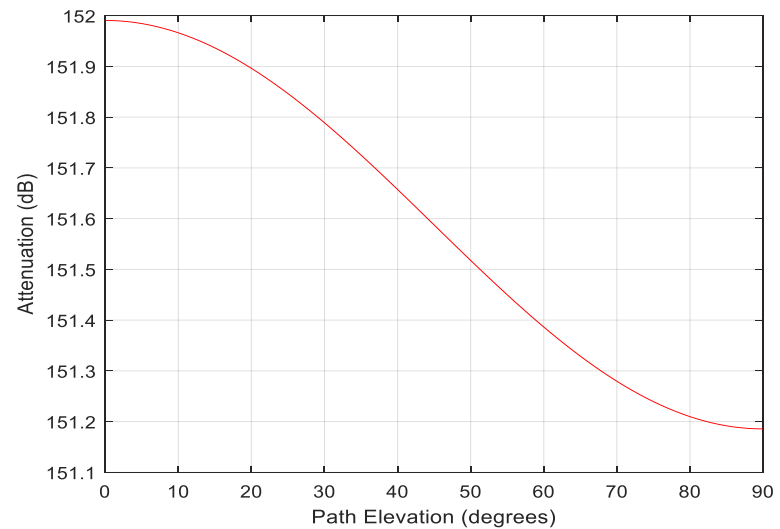

Fig.9 Signal attenuation due to rainfall as a function of elevation angle with rain rate is $10.00 \mathrm{~mm} / \mathrm{h}$ 
International Journal of Wireless \& Mobile Networks (IJWMN) Vol. 12, No. 3, June 2020

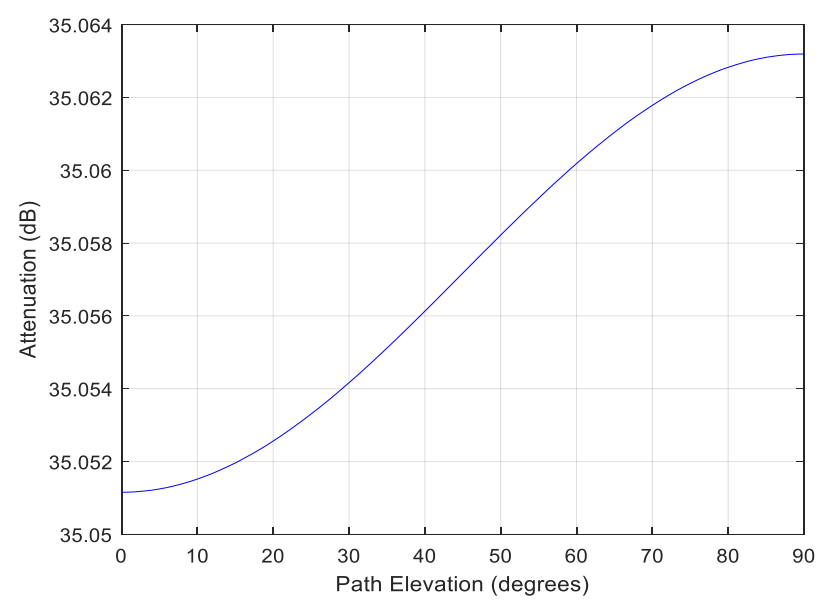

Fig.10 Signal attenuation due to rainfall as a function of elevation angle with rain rate $1.0 \mathrm{~mm} / \mathrm{h}$

Fig.11 and Fig.12 shows that signal attenuation due to rainfall as a function of horizontal polarization (elevation angle ' 0 ') with transmitted frequency $100 \mathrm{GHz}$ and rain rates are 1.00 $\mathrm{mm} / \mathrm{h}$ and $10.00 \mathrm{~mm} / \mathrm{h}$ respectively. It is clearly observed that $35.07 \mathrm{~dB}$ signal attenuation is obtained when tilt angles were $-62^{\circ}$ to $62^{\circ}$ and rain rate was $1.00 \mathrm{~mm} / \mathrm{h}$. At the same time 150.8 $\mathrm{dB}$ signal attenuation is obtained with the same tilt angles but rain rate was $10.00 \mathrm{~mm} / \mathrm{h}$.

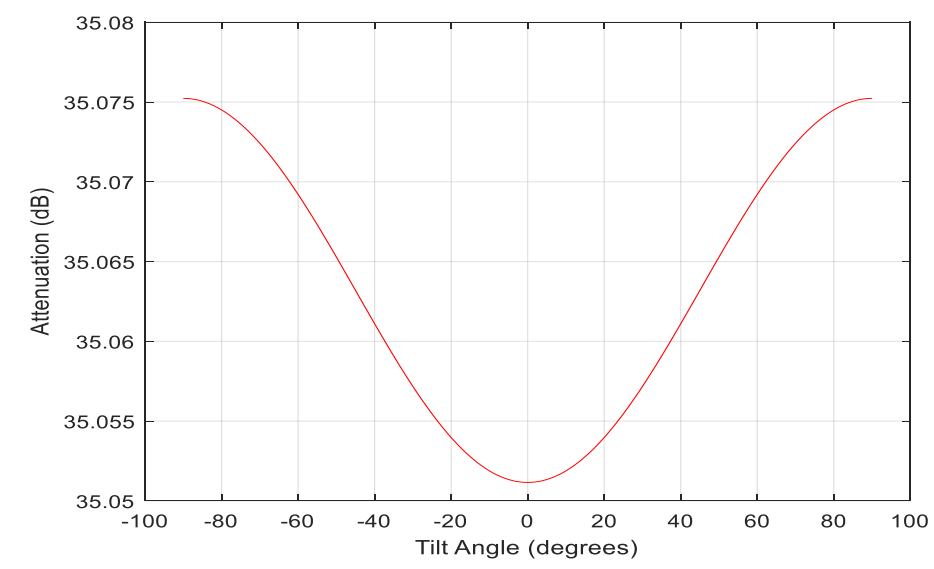

Fig.11 Signal attenuation due to rainfall as a function of horizontal polarization (rain rate $1.00 \mathrm{~mm} / \mathrm{h}$ )

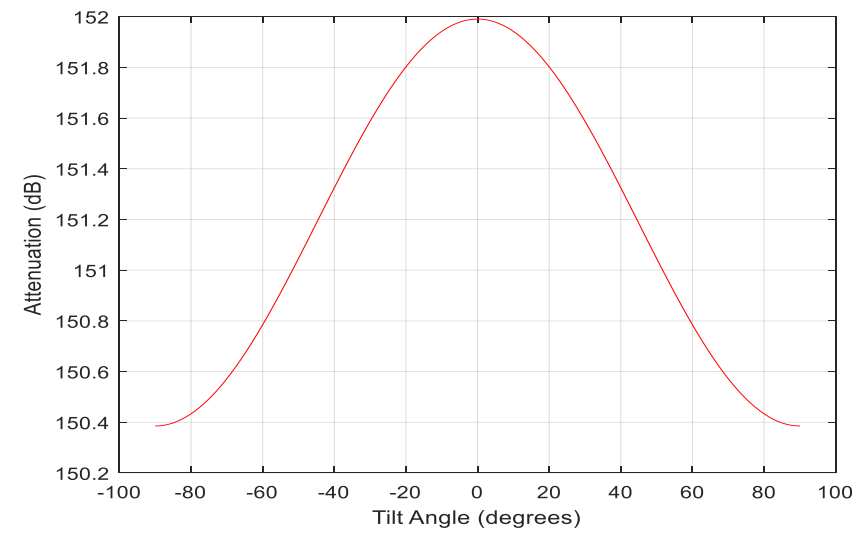

Fig.12 Signal attenuation due to rainfall as a function of horizontal polarization (rain rate $10.00 \mathrm{~mm} / \mathrm{h}$ ) 
International Journal of Wireless \& Mobile Networks (IJWMN) Vol. 12, No. 3, June 2020

Fig.13 illustrate that the signal propagation loss increases with increasing the frequency and range.

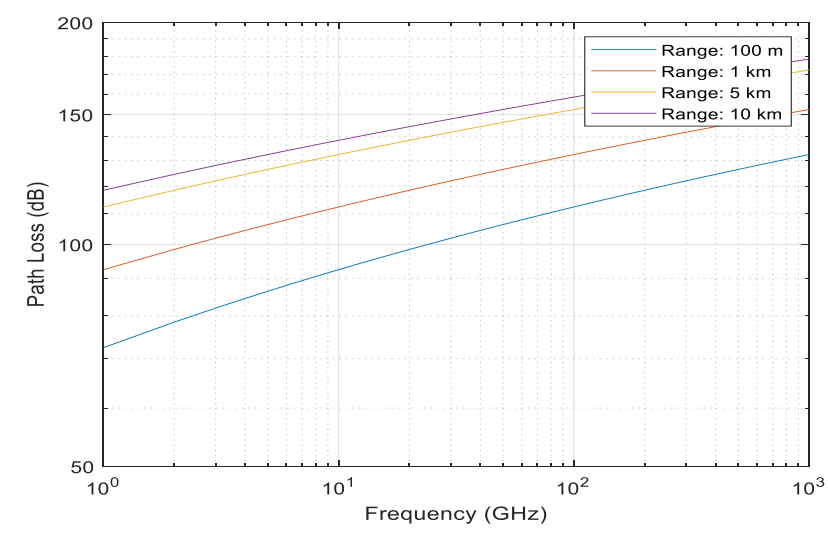

Fig.13 Free space path loss

Free space path loss for $77 \mathrm{GHz}$ automotive radar is shown in Fig.14. A comparison is shown considering all weather related for radar, named $77 \mathrm{GHz}$ automotive. Here free space path loss (FSPL) is the dominant loss. Losses due to fog, gas and rain are minor up to $100 \mathrm{GHz}$.

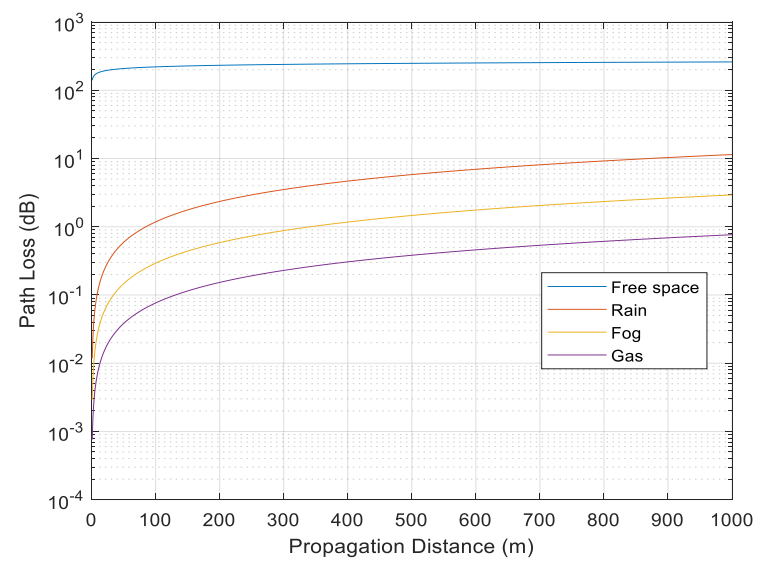

Fig.14 Free space path loss for $77 \mathrm{GHz}$ automotive radar

Fig. 15 shows how loses due to rain changes with frequencies. According to the ITU-model, rain that is characterized by the rain rate $(\mathrm{mm} / \mathrm{h})$. Rain is the main limiting factor particularly when operating above $5 \mathrm{GHz}$ for a radar system. Rain is classified on the basis of rain rate, namely: light rain $(1.0 \mathrm{~mm} / \mathrm{h})$, moderate rain $(4.0 \mathrm{~mm} / \mathrm{h})$, heavy rain $(10.0 \mathrm{~mm} / \mathrm{h})$ and extreme rain $(50.0$ $\mathrm{mm} / \mathrm{h})$. By the figure, it is observed that $11 \mathrm{~dB}$ attenuated for the extreme rain at the frequency $100 \mathrm{GHz}$ signal, where $1.01 \mathrm{~dB}, 2.02 \mathrm{~dB}$ and $7 \mathrm{~dB}$ attenuated for the light rain, moderate rain and heavy rain with the same frequency. 


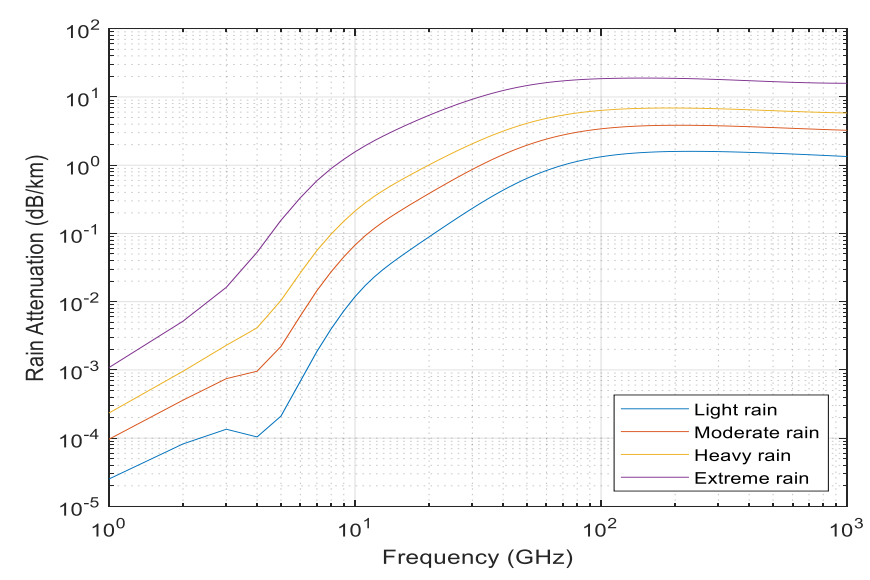

Fig.15 Attenuation due to rain

Fig.16 shows the signal propagation loss due to fog varies with frequencies. At $100 \mathrm{GHz}$ attenuations is negligible due to medium fog (visibility drops to approximately 300 meters and has a liquid water density (LWD) of $0.05 \mathrm{~g} / \mathrm{m}^{3}$ ). On the contrary, $5 \mathrm{~dB}$ attenuation is obtained due to heavy fog (visibility drops to roughly 50 meters and has a liquid water density $0.5 \mathrm{~g} / \mathrm{m}^{3}$ ). If angle of elevation is higher and smaller the attenuation then higher the value of $E_{b} / N_{0}$. During heavy rain considering about $0.001 \%$ of annual time, poor channel quality exerts a major problem to the subscribers of the networks for satellite wireless communication. For this, the link for the communication goes to outage at lower elevation angle.

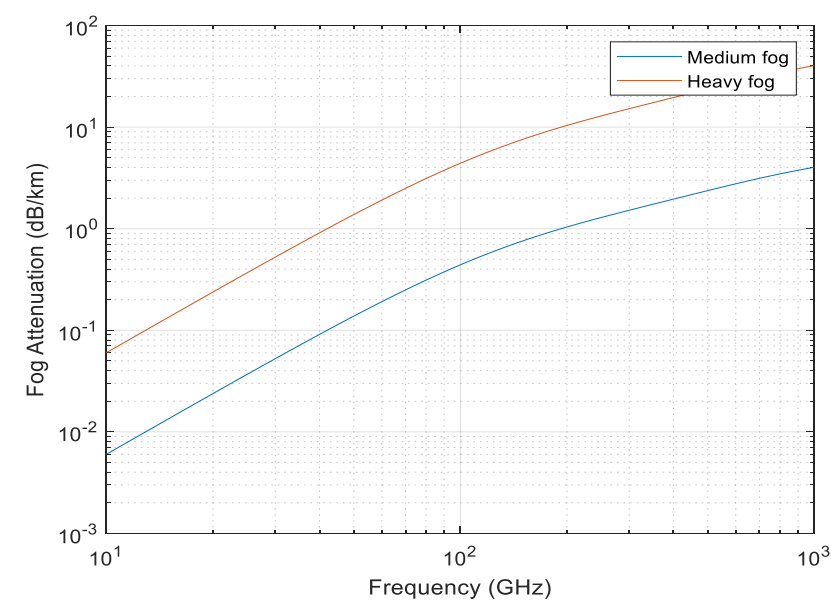

Fig.16 Attenuation due to fog

For calculating BER, mainly rely on the $E_{b} / N_{0}$ with a bandwidth having the bit rate for transmission. Fig.17 shows the BER for having different elevation angle of the modulation methods, QPSK, 8-PSK and 16-PSK, respectively. The occurrences in rainy weather, the upper M-array modulation system, BER will be higher owing to higher the transmitted bit in unit time. As a result, the angle of elevation is inversely proportional to BER. 


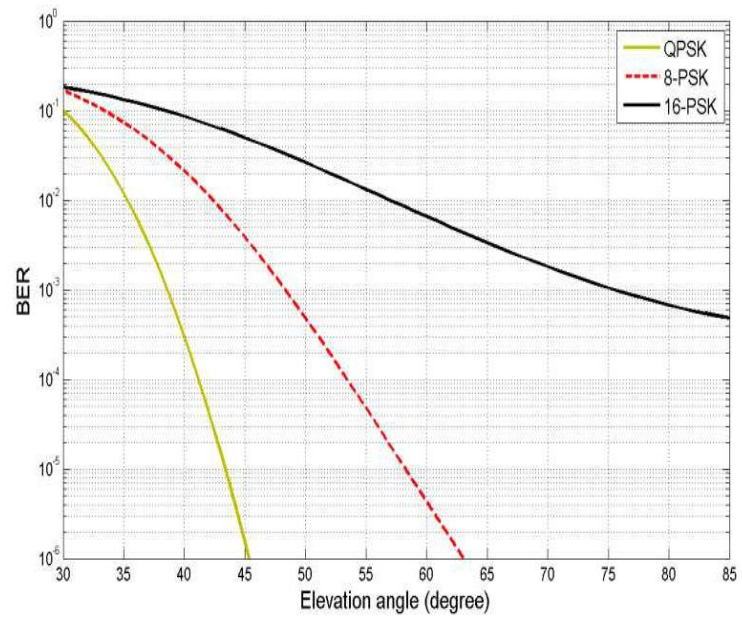

Fig.17. Bit Error Rate [9]

\section{CONCLUSION}

This paper present the effects of atmospheric impairments for different frequencies wireless communications signal. Different attenuation is called transmission impairments has been demonstrated caused by rain, clouds, gases and vapor of water. The findings of the atmospheric impairments were received on the real world actual measured parameters. For analyzing the performance, it was described by the use of different propagation modules for the wireless satellite radar communication including the various transmission parameters. From the results obtained it has shown that the attenuation effect due to rainstarted at the frequency band above $10 \mathrm{GHz}$ and showed the massive impact along with other atmospheric phenomena preceded by attenuation of the cloud and attenuation of the less effective gases. It is introduced atmospheric propagation model through this paper. The simulation results reveal that the frequency to be transmitted, the rate of rainfall, liquid water contents and relative humidity are directly proportional to the degradation of signal quality whereas angle of elevation is inversely proportional.

\section{ACKNOWLEDGEMENT}

The authors would like to thank to Department of Information and Communication Technology, Islamic University, Kushtia-7003, Bangladesh, center for advanced wireless communication research group for their unparalleled support to complete this work.

\section{REFERENCES}

[1] J. Mandeep and Y. Ng, "Satellite Beacon Experiment for Studying Atmospheric Dynamics,"Journal of Infrared, Millimeter and Terahertz Waves,vol. 31, pp. 988-994, 2010.

[2] Theodore S. Rappaport, Yunchou Xing, George R. MacCartney, Evangelos Mellios and Jianhua Zhang "Overview of Millimeter Wave Communications forFifth-Generation (5G) Wireless Networks-With a Focus on Propagation Models" IEEE TRANSACTIONS ON ANTENNAS AND PROPAGATION, VOL. 65, NO. 12, DECEMBER 2017, pp. 6213-6230.

[3] M. Kamruzzaman, M. S. Islam, "Rain Attenuation Prediction for Satellite Communications Link at $\mathrm{Ku}$ and Ka Bands Over Bangladesh" International Conference on Electrical Engineering and Information \& Communication Technology (ICEEICT) 2014, pp.1-6.

[4] Sujan Shrestha and Dong-You Choi, "Rain Attenuation Study over an $18 \mathrm{GHz}$ Terrestrial Microwave Link in South Korea" International Journal of Antennas and Propagation, March 2019. Volume 2019, 16 pages. 
International Journal of Wireless \& Mobile Networks (IJWMN) Vol. 12, No. 3, June 2020

[5] A. Adhikari, A. Bhattacharya, and A. Maitra, "Rain-Induced Scintillations and Attenuationof KuBand Satellite Signals at a Tropical Location," Geoscience and RemoteSensing Letters, IEEE, vol. 9, pp. 700-704, 2012.

[6] M. Zubair, Z. Haider, S. A. Khan, and J. Nasir,"Atmospheric influences on satellite communications,"Przeglad Elektrotechniczny, vol. 87, pp. 261-264, 2011.

[7] A. D. Panagopoulos, P. D. M. Arapoglou, and P. G. Cottis, "Satellite communicationsat KU, KA, and V bands: Propagation impairments and mitigation techniques," CommunicationsSurveys \& Tutorials, IEEE, vol. 6, pp. 2-14, 2004.

[8] T. V. Omotosho and C. O. Oluwafemi, "Impairment of radio wave signal by rainfallon fixed satellite service on earth-space path at 37 stations in Nigeria," Journal of Atmosphericand Solar-Terrestrial Physics, vol. 71, pp. 830-840, 2009.

[9] A.M. Al-Saegh, A. Sali, J.S. Mandeep and A Islamil," Extended atmospheric impairments on earthsky signal quality in tropical regions at Ku-band", Journal of Atmospheric and Solar-Terrestrial Physics, Vol.104, pp. 96-105, 2013.

[10] COST 255, "Radiowave Propagation Modeling For New Satcom Services At Ku-Band and above," Final Document. 2002.

[11] R. K. Crane, "Prediction of Attenuation by Rain,"Communications, IEEE Transaction, vol.28, pp.1717-1733, Sep 1980.

[12] I. T. U. ITU, "Propagation data and prediction methods required for the design of Earth-space telecommunication systems.," ed: International Telecommunication Union-Recommendation P.61811, 2013.

[13] ITU,"Characteristicsof precipitation for propagation modelling.,"ed:International Telecommunication Union-Recommendation P.837-6, 2012.

[14] A. Dissanayake, J. Allnutt, and F. Haidara, "A prediction model that combines rain attenuation and other propagation impairments along Earth-satellite paths," Antennasand Propagation, IEEE Transactions on, vol. 45, pp. 1546-1558, 1997.

[15] W. L. Stutzman and W. K. Dishman, "A simple model for the estimation of rain-induced attenuation along earth-space paths at millimeter wavelengths," Radio Sci., vol.17, pp. 1465-1476, 1982.

[16] S. D. A. Adhikari, A. Bhattacharya, and A. Maitra, "Improving rain attenuation estimation:modelling of effective path length using ku-band measurements at a tropical location," Progress In Electromagnetics Research B, vol. 34, pp. 173-186, 2011.

[17] Guide to Meteorological Instruments and Methods of Observation, World Meteorological Organization (WMO), Geneva, Switzerland, 2008.

[18] Gustavo A. Siles, Joes Mauel Riera and Pedro, "Atmospheric Attenuation in Wireless Communication Systems at Millimeter and THz Frequencies", IEEE Antennas and Propagation Magazine, Vol. 57, No. 1, February 2015, pp. 48-61.

[19] E. Salonen and S. Uppala, "New prediction method of cloud attenuation," Electronics Letters, vol. 27, pp. 1106-1108, 1991.

[20] I. T. U. ITU, "Attenuation due to clouds and fog.," ed: International Telecommunication UnionRecommendation P.840, 2012.

[21] A. Dissanayake, J. Allnutt, and F. Haidara, "Cloud attenuation modelling for SHF and EHF applications," International Journal of Satellite Communications, vol. 19, pp.335-345, 2001.

[22] E. E. Altshuler and R. A. Marr, "Cloud attenuation at millimeter wavelengths," Antennas and Propagation, IEEE Transactions on, vol. 37, pp. 1473-1479, 1989.

[23] L. J. Ippolito, Satellite communications systems engineering: atmospheric effects, satellite link design and system performance: Wiley, 2008.

[24] R. Bhattacharya, R. Das, R. Guha, S. D. Barman, and A. B. Bhattacharya, "Variability of millimetrewave rain attenuation and rain rate prediction: A survey," Indian Journalof Radio \& Space Physics (IJRSP), vol. 36, pp. 325-344, Aug-2007 2007.

[25] A. Maitra and S. Chakraborty, "Cloud Liquid Water Content and Cloud Attenuation Studies with Radiosonde Data at a Tropical Location," Journal of Infrared, Millimeterand Terahertz Waves, vol. 30, pp. 367-373, 2009.

[26] I. T. U. ITU, "Attenuation by atmospheric gases.," International Telecommunication UnionRecommendation P.676-10, 2013.

[27] I.T.U. ITU-R, "Attenuation by atmospheric gases and related effects", ed: International Telecommunicatio Union- Recommendation P.676-12, 2019. 
[28] L. Castanet, M. Bousquet, and D. Mertens, "Simulation of the performance of a Kaband VSAT videoconferencing system with uplink power control and data rate reduction to mitigate atmospheric propagation effects," International Journal of SatelliteCommunications, vol. 20, pp. 231-249, 2002.

[29] J. S. Mandeep, S. I. S. Hassan, and K. Tanaka, "Rainfall measurements at Ku-band satellite link in Penang, Malaysia," Microwaves, Antennas \& Propagation, IET, vol. 2, pp. 147-151, 2008.

\section{AUTHORS}

Sharmin Tajria Ananya received the B.Sc.(Hons). degree from the Department of Information and Communication Technology, Islamic University, Kushtia-7003, Bangladesh in 2016. Currently, she is a student of M.Sc. (Thesis group) of the same department and also waiting to receive the final result. Her research interests are Image processing and Wireless communications.

Md. Shariful Islam received the B.Sc.(Hons). and M.Sc. degrees from the Department of Electrical and Electronic Engineering (Erstwhile of Applied Physics, Electronics and Communication Engineering) from Islamic University, Kushtia-7003, Bangladesh, in 1999(Examination held 2001) and 2000 (Examination held-2003), respectively. He also received the $\mathrm{PhD}$ degree in 2016 from Islamic University, Kushtia-7003, Bangladesh. He joined as a lecturer in the Dept. of Information and Communication Technology, IU,Kushtia-7003, Bangladesh, in September 2004 and currently, he is working as Professor in the same department. His research interests are in the area of Integral Imaging 3D display technology.

Image processing, Wireless Communications

Md. Ashek Raihan Mahmud received the B.Sc. (Engg.) from the Department of Electrical and Electronic Engineering from Rajshahi University of Engineering and Technology, Rajshahi, Bangladesh. He also received M.Sc. and M.Phil. from Australia (RMIT) as well as Islamic University, Kushtia-7003, Bangladesh. He joined as a lecturer in the Dept. of Information and Communication Technology, IU,Kushtia-7003, Bangladesh, in September 2004 and currently, he is working as an Associate Professor in the same department. His research interests are in the area

of Mobile Ad-hoc Network, Telecommunication.

Dr. Pallab Podder (S'14 M'18) has completed his PhD from the CSU Machine Learning Research Unit of Charles Sturt University, Australia in December-2017. Previously he completed Masters (M.Sc.) and B.Sc (Hons) degree and from the department Information \& Communication Engineering in 2010 and 2008 respectively. After his M.Sc. degree, he joined as a lecturer in the Computer Science \& Engineering Department of Bangladesh University, Dhaka, Bangladesh. Then he joined as a lecturer and promoted to Assistant Professor in the Department of Information \&
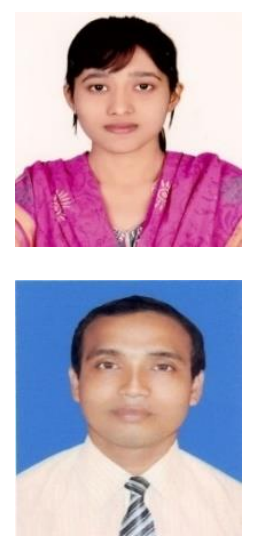
Communication Engineering of Pabna Science \& Technology University, Pabna, which is one of the renowned universities in Bangladesh. After that, he has been serving as an Associate Professor and Chairman of the Information and Communicaton Engineering Department of the same University since 18 August 2018 and 28 September 2019 respectively. He has published more than 30 journal articles and International conference proceedings in the areas of communication, image processing, video compression, and video quality assessment. He is a member of the IEEE, Bangladesh Computer Council (BCC), and ACS (Australian Computer Society).

Md. Jashim Uddin received the B.Sc. (Honors) and M.Sc. from the Department of Information and Communication Technology, Islamic University, Kushtia-7003, Bangladesh in 2005 and 2006 respectively. Presently, he is a PhD student on IoT. He joined as a lecturer in the Department of Information and Communication Technology, Islamic University, Kushtia-7003, Bangladesh in 2010 and currently he is working as an Assistant Professor in the same department. His research interests are in the area of Internet of Things (IoT), Image and Speech Processing.

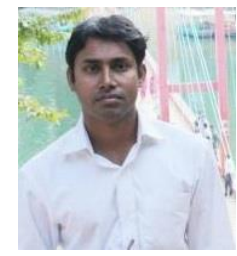

